\title{
Impact of socioeconomic disparities on cause-specific survival of retinoblastoma
}

\author{
REX CHEUNG
}

Bryn Mawr, PA 19010, USA

Received December 7, 2012; Accepted February 27, 2013

DOI: $10.3892 / \mathrm{mco} .2013 .83$

\begin{abstract}
Retinoblastoma (RB) is a rare disease of infancy and early childhood. This study investigated the effects of socioeconomic factors on the cause-specific survival of RB. Data from patients diagnosed with RB between 1973 and 2009 were obtained from the Surveillance, Epidemiology and End Results (SEER) database. The study included 1,456 patients with a the mean follow-up time (SD) of 128.75 (113.74) months and a mean age (SD) of 1.4 (2.6) years. This study analyzed socioeconomic, staging and treatment factors available in the SEER database for RB. Kaplan-Meier analysis was used to analyze time-to-failure data. The two-sample Kolmogorov-Smirnov test was used for univariate analysis and the Cox proportional hazards model was used for multivariate analysis. The area under the receiver operating characteristic (ROC) curve was computed for predictors. SEER stage was the most significant predictive pretreatment factor. The identified socioeconomic barriers included ethnicity and rural-urban residence status that led to a $3 \%$ decrease in RB cause-specific survival. Thus, eliminating barriers to treatment is crucial for reducing the outcome disparities.
\end{abstract}

\section{Introduction}

Retinoblastoma (RB) is a rare disease of infancy and early childhood (1). It is rarely encountered in adults. The majority of RB patients exhibit excellent survival outcomes $(2,3)$. At present, the standard staging system that is internationally used is the International Retinoblastoma Staging System (4). Staging is an important factor affecting treatment selection and outcome (5). Previous studies demonstrated that African-American descent adversely affected the outcome of RB, a finding that may be due to limited access to treatment $(6,7)$. Further investigations are required to identify the socioeconomic barriers to optimal RB outcomes.

The Surveillance, Epidemiology and End Results (SEER; http://seer.cancer.gov/) program is a public-use cancer registry

Correspondence to: Dr Rex Cheung, 275 S South Bryn Mawr Ave., K43, Bryn Mawr, PA 19010, USA

E-mail: cheung.r100@gmail.com

Key words: Surveillance, Epidemiology and End Results database, retinoblastoma, socioeconomic factors, cause-specific survival of the USA National Cancer Institute. SEER is widely used as a source of benchmark data for studying RB outcomes in the USA as well as in other countries (1,3,8-11). In addition to the biological and treatment factors, this database also provides a large number of county-level socioeconomic factors. This study was part of a larger study that aimed to identify barriers to optimal cancer treatment outcomes, which may be discernable only from information obtained from a national database.

\section{Materials and methods}

SEER is a public-use database that may be used for analysis with no requirement for internal review board approval. SEER Clinical Outcome Prediction Expert (SCOPE) (12) was used to mine SEER data and construct accurate and efficient prediction models $(13,14)$. Data were obtained from the SEER 18 database, using the filter 'Site and Morphology'. ICCC site recode ICD-O-3 = 'V Retinoblastoma'. The SEER'Stat statistical software (http://seer.cancer.gov/seerstat/) was used for case listing. Kaplan-Meier analysis was used to assess the time to RB-specific mortality (coded as Eye and Orbit mortality in SEER) data. The two-sample Kolmogorov-Smirnov test was used to assess the significance of the difference between two survival curves. The Cox proportional hazards model was used for multivariate analysis. For univariate and multivariate analyses, coding was as follows: i) SEER stage: 0, local/regional; 1, metastatic/unstaged; ii) county-level rural vs. urban residence status: 0 , urban residence; 1 , rural residence; iii) race/ethnicity: 0, non-African American; 1, African American; iv) county-level percentage of college graduates: $0,>25 \%$; $1, \leq 25 \%$; v) county-level household income: 0, >55,000 USD/year; 1, $\leq 55,000$ USD/year. All statistics and programming were performed by Matlab (www. mathworks.com). The areas under the receiver operating characteristic (ROC) curves were computed for predictors. In addition, binary fusion and optimization were used to streamline the ROC risk stratification by combining risk strata when possible. Similar strata were fused to create more efficient models if the resultant ROC performance did not degrade $(13,14)$.

\section{Results}

A total of 1,456 patients were included in this study (Table I). The Kaplan-Meier survival curve exhibited an excellent 
Table I. Univariate risk models including sociodemographic, tumor and treatment risk factors for disparity in RB treatment outcome.

\begin{tabular}{lc}
\hline Initial univariate risk models & No. \\
\hline Study population & 1,456 \\
Gender &
\end{tabular}

Gender

Male

Female

Mean follow-up time in months (SD)

Mean age of diagnosis in years (SD)

Patient age (years)

$\geq 20$

$<20$

Race and ethnicity

White

Other $^{\mathrm{a}}$

Black

Unknown

Other unspecified (1991+)

Radiation treatment

Beam radiation

Combination of beam with implants or isotopes

Radioactive implants

None

Recommended, unknown if administered

Radiation, NOS method or source not specified

Unknown

Refused

Radioisotopes

Surgery recommendations

Reasons other than cancer

Surgery performed

Recommended but not performed, unknown reason

Unknown; death certificate or autopsy only case

Not recommended, contraindicated due to other conditions

Not recommended

Recommended, unknown if performed

Recommended but not performed, patient refused

County-level annual household income

$\geq 55,000$ USD

$<55,000$ USD

County-level \% college graduates

$\geq 25 \%$

$<25 \%$

Rural-urban continuum code 2003

Counties in metropolitan areas, $250,000-1$ million pop

Counties in metropolitan areas $\geq 1$ million pop

Urban pop of 2,500-19,999, not adjacent to a metro area

Urban pop of ge 20,000 adjacent to a metropolitan area

Counties in metropolitan areas of $<250,000$ pop

Comp rural $<2,500$ urban pop, adjacent to a metro area

Urban pop of 2,500-19,999, adjacent to a metro area

Comp rural $<2,500$ urban pop, not adjacent to metro area

Urban pop of $\geq 20,000$ not adjacent to a metropolitan area

Unknown/missing/no match (Alaska - Entire State)
760

696

52.16

47.77

$\%$

Model

ROC

SD

128.75 (113.14)

1.4 (2.6)

$\begin{array}{cr}5 & 0.34 \\ 1,451 & 99.66\end{array}$

1,069

73.37

10.71

14.21

1.30

0.34

183

1
21

1,217

13

8

11

1

1

12.56

0.07

1.44

83.53

0.89

0.55

0.75

0.07

0.07

1

1,248

76

0.07

85.66

5.22

1.17

0.07

7.69

0.07

0.07

42.24

57.76

50.07

49.93 
Table I. Continued.

\begin{tabular}{lcccc}
\hline Initial univariate risk models & No. & $\%$ & Model & ROC \\
\hline SEER historical stage A & & & I, II, III, IV & 0.01 \\
Localized, I & 1,040 & 71.38 & optimized & 0.64 \\
Regional, II & 185 & 12.70 & I, (II, III), IV & 0.64 \\
Distant, III & 119 & 8.17 & \\
Unstaged, IV & 112 & 7.69 & & \\
COD to site rec KM & & & \\
Alive & 1,362 & 93.48 & \\
Eye and Orbit & 49 & 3.36 & \\
Others & 45 & 3.09 & \\
\hline
\end{tabular}

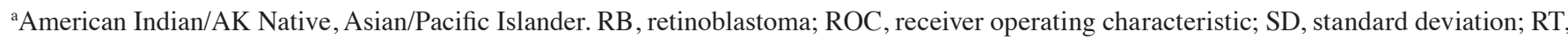
radiotherapy; pop, population; Comp rural, completely rural; COD to site rec KM, cause of death to SEER site record Kaposi sarcoma and mesothelioma.

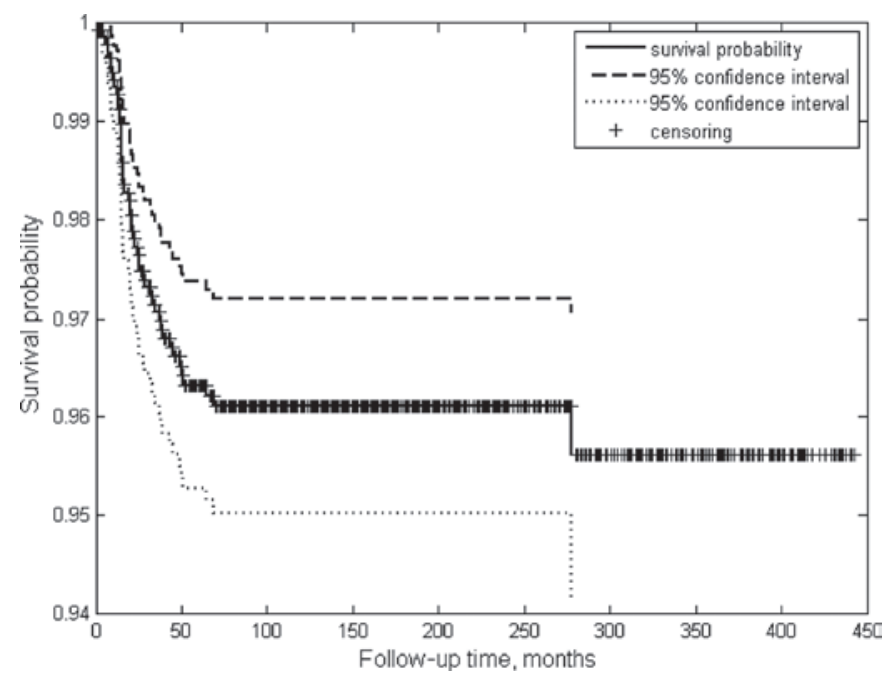

Figure 1. Kaplan-Meier analysis of time to retinoblastoma-related mortality.

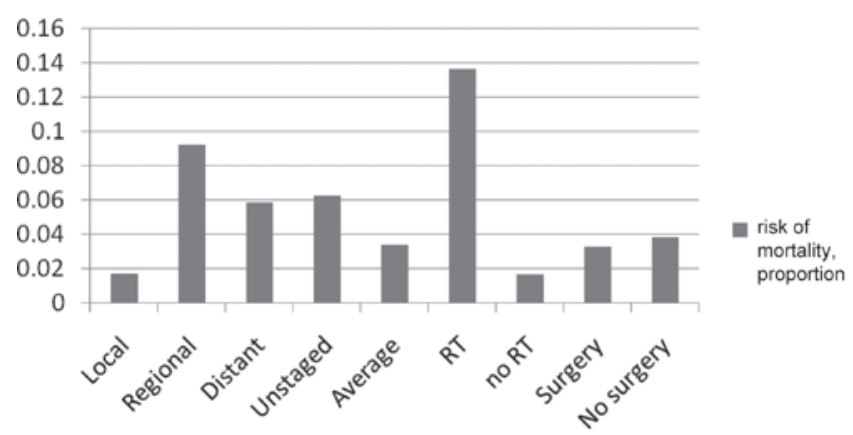

Figure 2. Risk of cause-specific mortality by the Surveillance, Epidemiology and End Results (SEER) stage and by treatment choice (radiation or surgery). Mortality was higher in RT compared with RT, surgery and no surgery groups. $\mathrm{RT}$, radiation treatment.

long-term cause-specific survival rate of $>90 \%$ (Fig. 1). The mean follow-up time (SD) was 128.75 (113.74) months and the mean age (SD) was 1.4 (2.6) years. There were only 5 adult RB patients listed by the SEER 18 database, a number representing
Table II. Risk of RB-specific mortality (\%) associated with gender, age and various socioeconomic models.

\begin{tabular}{lcc}
\hline Predictors & Patient no. & $\%$ mortality \\
\hline Gender & & \\
Female & 696 & 0.03 \\
$\quad$ Male & 760 & 0.03 \\
Age (years) & & \\
$\quad<20$ & 1,451 & 0.03 \\
$\geq 20$ & 5 & 0.00 \\
County \% college graduates & & \\
$>25 \%$ & 729 & 0.03 \\
$\quad \leq 25 \%$ & 727 & 0.03 \\
Rural-urban & & \\
continuum code 2003 & & 0.03 \\
Metropolitan & 1,313 & 0.06 \\
No & 143 & \\
County-level annual & & 0.03 \\
household income & & \\
$\geq 55,000$ USD & & 0.03 \\
$<55,000$ USD & 615 & \\
Ethnicity & & \\
African-American & & \\
Others & & \\
\hline
\end{tabular}

$\mathrm{RB}$, retinoblastoma.

28\% of the USA cases reported between 1973 and 2009. The majority of the patients had been staged and SEER stage was the most significant predictive factor, with an ROC area of 0.64 (0.01) (Table I). The ROC area of this model was computed from 5 samples that were randomly selected from the case pool. Each sample represented $50 \%$ of the total number of cases. As shown in Fig. 2, the risk did not progress from lower (local stage)- to higher (distant stage)-risk groups. Furthermore, 
A
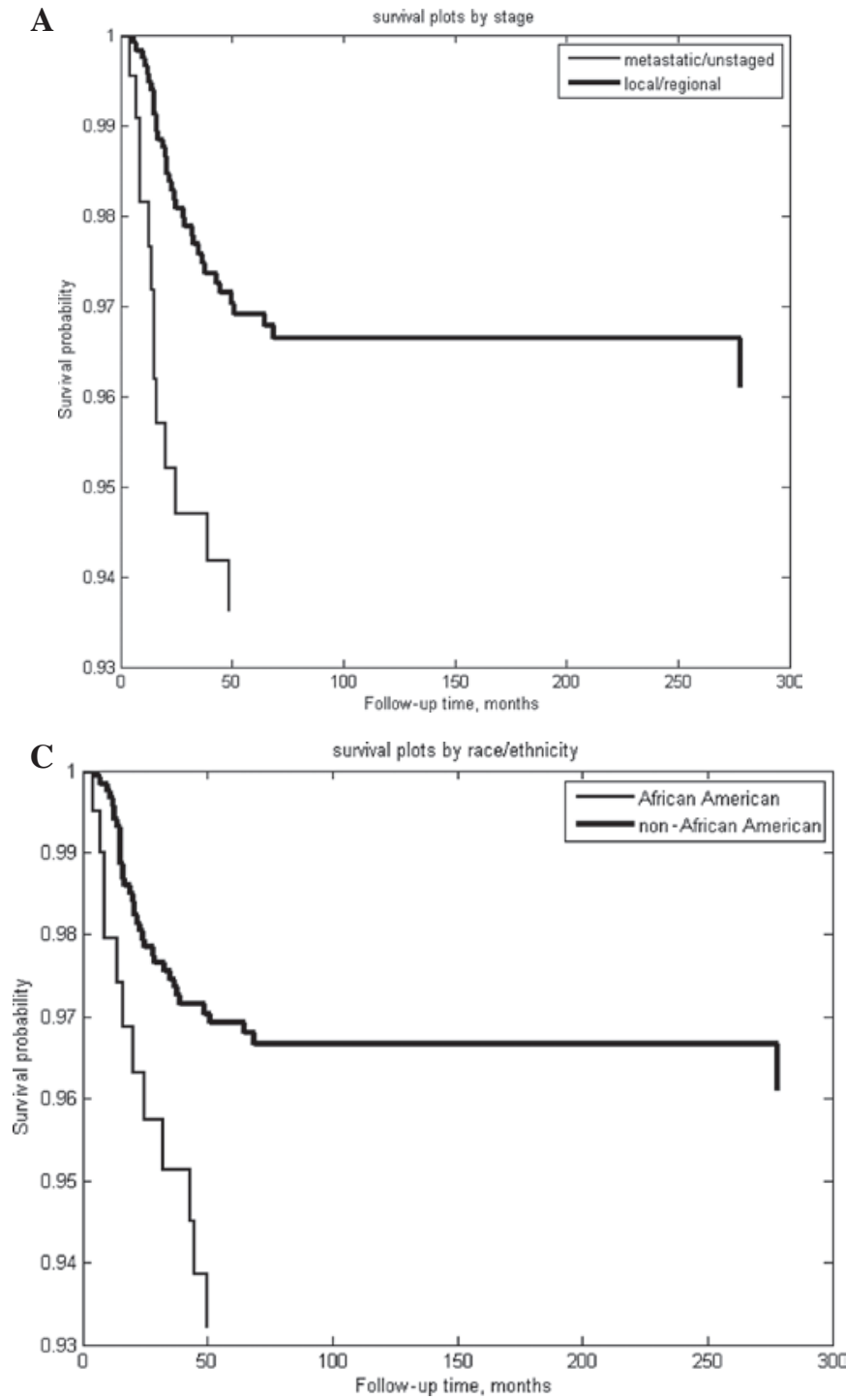

B

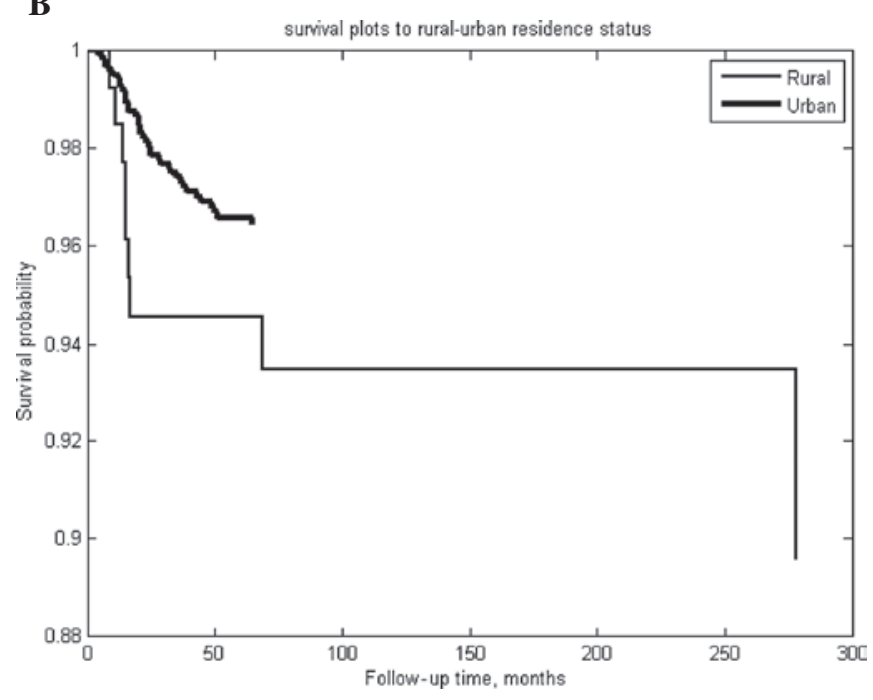

D survival plots by county \% college graduate

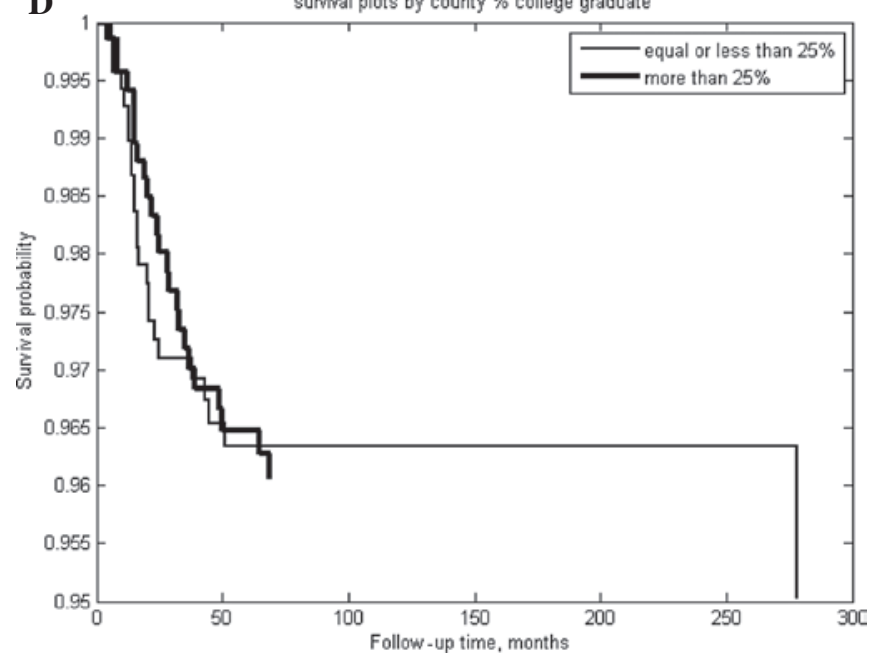

$\mathbf{E}$

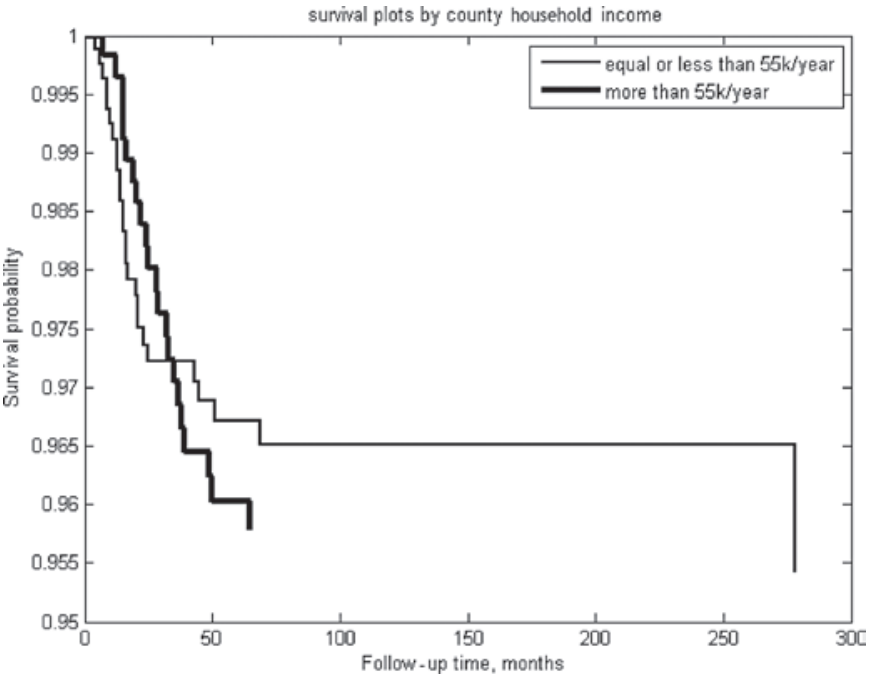

Figure 3. Kolmogorov-Smirnov tests. (A) Surveillance, Epidemiology and End Results (SEER) stage, (B) rural-urban residence status, (C) race/ethnicity, (D) county-level percentage college graduates and (E) county-level household income.

ROC analysis revealed that regional and distant groups may be combined in terms of predicting the cause-specific survival of RB patients. A significant number of unstaged patients were identified, accounting for $7.5 \%$ of the patient population
(Table I and Fig. 2). Unstaged patients exhibited a high risk of mortality, comparable to that of metastatic RB patients (Fig. 2). The SEER staging model was initially created as a 4-tiered model (Table I). Radiotherapy (RT) was used in $~ 10 \%$ 
Table III. Univariate and multivariate analyses of RB prognosticators.

\begin{tabular}{|c|c|c|c|c|c|c|}
\hline \multirow[b]{2}{*}{ Predictors } & \multicolumn{3}{|c|}{ Kolmogorov-Smirnov test } & \multicolumn{3}{|c|}{ Cox proportional hazard model } \\
\hline & $\mathrm{h}$ & P-value & $\kappa$ & $\beta$ & SE & P-value \\
\hline SEER stage & & & & & & \\
\hline $\begin{array}{l}0, \text { local/regional } \\
1, \text { metastatic/unstaged }\end{array}$ & 1 & 0.0284 & 0.4688 & 0.7737 & 0.3166 & 0.0145 \\
\hline $\begin{array}{l}\text { Rural-urban residence } \\
0, \text { urban residence } \\
1, \text { rural residence }\end{array}$ & 1 & 0.0152 & 0.5556 & 0.9337 & 0.4008 & 0.0198 \\
\hline $\begin{array}{l}\text { Race/ethnicity } \\
0, \text { non-African-American } \\
1 \text {, African-American }\end{array}$ & 1 & 0.0234 & 0.4833 & 0.804 & 0.3375 & 0.0172 \\
\hline $\begin{array}{l}\text { County \% college graduates } \\
0,>25 \% \\
1, \leq 25 \%\end{array}$ & 0 & 0.9879 & 0.134 & 0.0881 & 0.4123 & 0.8308 \\
\hline $\begin{array}{l}\text { County household income } \\
0,>55,000 / \text { year } \\
1, \leq 55,000 / \text { year }\end{array}$ & 0 & 0.7974 & 0.1905 & -0.3622 & 0.4151 & 0.383 \\
\hline
\end{tabular}

For two-sample Kolmogorov-Smirnov tests, h was 1 when the test was statistically significant for the $\kappa$ statistics. $\beta$ and SE were respectively the Cox proportional hazard coefficients and standard errors. $\mathrm{P}<0.05$ was considered significant. RB, retinoblastoma; SEER, Surveillance, Epidemiology and End Results.

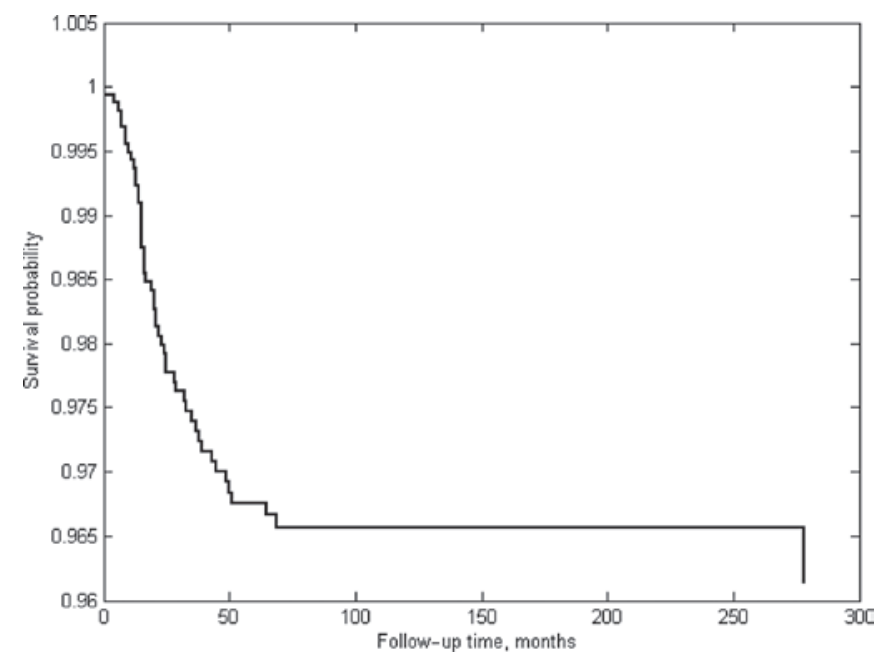

Figure 4. Cox proportional hazard fit of the model shown in Table III.

of patients and was predictive of worse outcome (RB-specific mortality risk, 13.7\%). The use of RT as an eye-preservation treatment has declined over the years (Fig. 3), possibly due to the secondary cancers that have been attributed to RT in RT-treated patients (15).

As regards the pretreatment factors, Table II shows that gender, county-level household income and county-level percentage of college graduates did not divide RB patients into subgroups with distinct risk factors of cause-specific RB mortality. The mean follow-up time was $\sim 10$ years (Table I) and the overall risk of cause-specific mortality was $\sim 3 \%$
(Fig. 2). However, groups not optimal in terms of race and rural-urban continuum factors have doubled this risk to $\sim 6 \%$. Thus, race and rural-urban continuums were expected to exhibit large ROC areas. However, their ROC areas were only moderately larger than the expected 0.5 for a random variable (Table I). When analyzed by time to cause-specific mortality, however, Fig. 3 and Table III show that SEER stage, race and rural-urban residence status were significant univariate predictors, unlike county-level household income or percentage of college graduates. Table III shows that the effects of race and rural-urban residence on $\mathrm{RB}$ outcome, as measured by Cox coefficients, are comparable to the effect of SEER stage. The Cox proportional hazard fit from the related Cox analysis (Table III) is shown in Fig. 4.

\section{Discussion}

RB treatment exhibits success rates of $>90 \%$ (Fig. 1). Previous studies demonstrated that socioeconomic factors may affect the outcome of RB patients $(6,7)$ and that relocation of individuals from low-income to higher-income neighborhoods lowered the rates of obesity and diabetes over a 10-15-year follow-up period $(16,17)$. The aim of this study was to identify socioeconomic factors affecting the cause-specific survival of $\mathrm{RB}$, in order to generate testable hypotheses for future trials of removing socioeconomic barriers to optimal RB outcomes. Therefore, this study investigated numerous possible explanatory factors (Table I).

The use of RT has declined over the years. This is likely due to severe long-term side effects. However, the long-term outcomes following treatment with aggressive chemotherapy 
have not been well characterized (2). Considering the improvement in proton therapy techniques, modern image guidance coupled with proton beam RT may need to be re-evaluated regarding its utility in the treatment of RB (18-20).

The International Retinoblastoma Staging System (4) serves as an important guide for the treatment selection and outcome of RB patients (5). However, this study used SEER staging, which has been consistent over the years, in order to analyze follow-up data in their entirety. SEER staging was identified as the most significant pretreatment predictive factor (Table I). After binary fusion, the optimized staging was reduced to a 3-tiered classification (Fig. 2 and Table I). Such efficient models may aid in reducing the number of patients required for clinical trials, since it has fewer risk groups to balance. Whether the SEER staging model is more accurate compared to the alternative models $(1,3)$ may be elucidated by further investigations. As a point of reference, we estimated that the ROC area of a commonly used prognostic model for prostate cancer using PSA, Gleason Score and prostate T-stage, had a ROC area of $0.75(13,14,21)$.

Using ROC area as a metric and a binary fusion algorithm, the 4-tiered SEER staging model was simplified into a 3-tiered model. The ROC area of this model was comparable to the original risk model. Thus, the model is simplified by $25 \%$ without an accuracy penalty. This may be of significance, considering that $25 \%$ less trial participants may be required to balance the risk profiles of the test and control arms. This is particularly relevant since several clinical trials are available for RB and other childhood cancers $(6,7)$. Unstaged patients are associated with a high risk of mortality, comparable to that of metastatic RB patients (Fig. 2), possibly due to the fact that without accurate staging, it would be difficult to select the optimal treatment option. Staged patients fared better compared to the overall cohort (Table II and Fig. 3A).

SEER data are particularly useful in ascertaining treatment individualization and have been used by previous studies $(7,9-11)$. In order to demonstrate the independent prognostic values of socioeconomic factors, we performed univariate and multivariate analyses of socioeconomic factors in combination with the most significant biological factor (SEER stage). Residing in areas with populations of $<25,000$ was associated with high risk of RB-specific mortality, as was African American descent (Table II, Fig. 3B-C and Table III); however, county-level household income and percentage of college graduates were not associated with a higher risk of mortality (Fig. 3D-E and Table III).

In conclusion, this study identified the most prognostic staging models according to pretreatment factors for RB cancer patients. Socioeconomic barriers identified included race and rural-urban residence. African American descent and rural residence led to a $3 \%$ decrease (Table II) in RB cause-specific survival. Eliminating barriers to optimal treatment may reduce outcome disparity in RB patients.

\section{References}

1. Broaddus E, Topham A and Singh AD: Incidence of retinoblastoma in the USA: 1975-2004. Br J Ophthalmol 93: 21-23, 2009.
2. Abramson DH, Marr BP, Brodie SE, et al: Ophthalmic artery chemosurgery for less advanced intraocular retinoblastoma: five year review. PLoS One 7: e34120, 2012.

3. Broaddus E, Topham A and Singh AD: Survival with retinoblastoma in the USA: 1975-2004. Br J Ophthalmol 93: 24-27, 2009.

4. Sastre X, Chantada GL, Doz F, et al: Proceedings of the consensus meetings from the International Retinoblastoma Staging Working Group on the pathology guidelines for the examination of enucleated eyes and evaluation of prognostic risk factors in retinoblastoma. Arch Pathol Lab Med 133: 1199-1202, 2009.

5. Schvartzman E, Chantada G, Fandino A, et al: Results of a stage-based protocol for the treatment of retinoblastoma. J Clin Oncol 14: 1532-1536, 1996.

6. Pui CH, Pei D, Pappo AS, et al: Treatment outcomes in black and white children with cancer: results from the SEER database and St Jude Children's Research Hospital, 1992 through 2007. J Clin Oncol 30: 2005-2012, 2012.

7. Liu L, Krailo M, Reaman GH and Bernstein L: Childhood cancer patients' access to cooperative group cancer programs: a population-based study. Cancer 97: 1339-1345, 2003.

8. Kleinerman RA, Tucker MA, Abramson DH, et al: Risk of soft tissue sarcomas by individual subtype in survivors of hereditary retinoblastoma. J Natl Cancer Inst 99: 24-31, 2007.

9. Lanier AP, Holck P, Ehrsam Day G and Key C: Childhood cancer among Alaska natives. Pediatrics 112: e396, 2003.

10. Gatta G, Capocaccia R, Coleman MP, Ries LA and Berrino F: Childhood cancer survival in Europe and the United States. Cancer 95: 1767-1772, 2002.

11. Breslow NE and Langholz B: Childhood cancer incidence: geographical and temporal variations. Int J Cancer 32: 703-716, 1983.

12. Cheung R: Poor treatment outcome of neuroblastoma and other peripheral nerve cell tumors may be related to under usage of radiotherapy and socio-economic disparity: a US SEER data analysis. Asian Pac J Cancer Prev 13: 4587-4591, 2012.

13. Cheung R, Altschuler MD, D'Amico AV, et al: ROC optimization may improve risk stratification of prostate cancer patients. Urology 57: 286-290, 2001.

14. Cheung R, Altschuler MD, D'Amico AV, et al: Using the receiver operating characteristic curve to select pretreatment and pathologic predictors for early and late postprostatectomy PSA failure. Urology 58: 400-405, 2001.

15. Vasudevan V, Cheung MC, Yang R, et al: Pediatric solid tumors and second malignancies: characteristics and survival outcomes. J Surg Res 160: 184-189, 2010.

16. Ludwig J, Sanbonmatsu L, Gennetian L, et al: Neighborhoods, obesity, and diabetes - a randomized social experiment. N Engl J Med 365: 1509-1519, 2011.

17. Ludwig J, Duncan GJ, Gennetian LA, et al: Neighborhood effects on the long-term well-being of low-income adults. Science 337: 1505-1510, 2012.

18. Chang JW, Yu YS, Kim JY, et al: The clinical outcomes of proton beam radiation therapy for retinoblastomas that were resistant to chemotherapy and focal treatment. Korean J Ophthalmol 25: 387-393, 2011.

19. Munier FL, Verwey J, Pica A, et al: New developments in external beam radiotherapy for retinoblastoma: from lens to normal tissue-sparing techniques. Clin Experiment Ophthalmol 36: 78-89, 2008.

20. DeLaney TF: Clinical proton radiation therapy research at the Francis H. Burr Proton Therapy Center. Technol Cancer Res Treat 6 (Suppl 4): 61-66, 2007.

21. Hanley JA and McNeil BJ: The meaning and use of the area under a receiver operating characteristic (ROC) curve. Radiology 143: 29-36, 1982. 